\title{
Differential expression and significance of Stat3 and IL-17 in gastritis and gastric intraepithelial neoplasia and gastric cancer
}

\section{Lijie Wang ( $\sim 641302483 @ q q . c o m$ )}

Shihezi University School of Medicine

Chong Cao

Shihezi University School of Medicine

Xiao Jun Wang

Shihezi University School of Medicine

Lin Tao

Shihezi University School of Medicine

\section{Feng Li}

Shihezi University School of Medicine

\section{Wen Jie Zhang}

Shihezi University School of Medicine

\section{Research}

Keywords: IL-17, Stat3, Gastritis, gastric intraepithelial neoplasia, Gastric cancer

Posted Date: February 10th, 2020

DOI: https://doi.org/10.21203/rs.2.22990/v1

License: (c) (i) This work is licensed under a Creative Commons Attribution 4.0 International License. Read Full License 


\section{Abstract}

Background: The role of Stat3 and IL-17 in the development of gastric cancer was investigated by detecting Stat3 activation level and IL-17 expression in gastritis, GIN and gastric cancer.

Methods: (1) We collected 70,137 patients who underwent gastroscopy at the First Affiliated Hospital of Shihezi University Medical School from November 2007 to December 2015, coded and screened all the diagnostic results. (2) We used immunohistochemical to detect differences in phosphorylated Stat3 and IL-17 expression in gastritis, GIN, and gastric cancer. (3) Kruskal-Wallis $\mathrm{H}$ test compared IL-17 or p-Stat 3 expression in gastric cancer, GIN and gastritis; Non-parametric test compared difference of IL-17 expression and $\mathrm{p}$-Stat3 activation level between randomized and disease progression groups indifferent gastric diseases.

Results: (1)The results of immunohistochemistry showed that as the degree of disease increased, the staining increased too; (2) There were differences in Stat3 activation level and IL-17 expression between gastritis, GIN and gastric cancer tissues $(P<0.001)$. With the disease increasingly serious, the Stat3 activation level and IL-17 expression are higher; (3) The ROC of p-Stat3 is the largest $(0.923, \mathrm{P}<0.001)$ and the ROC of IL-17 is $0.844(\mathrm{P}<0.001)$; (4) The activated Stat3 and IL-17 both are the risk factors of gastric cancer.

Conclusions: (1) With the disease increasingly serious, the Stat3 activation level and IL-17 expression are higher. (2) There is a high correlation between activated Stat3 and IL-17 expression, both of which are risk factors for gastric cancer.

\section{Background}

Gastric cancer (GC) is very common in China, which poses a threat to the public health. According to the Global Cancer Statistics 2018, an estimate of 456,124 new GC cases and 390,182 deaths occur in China, accounting for $44.1 \%$ and $49.9 \%$ of the world, respectively, and the morbidity and mortality rank second in malignant tumors [1,2]. In recent years, with the advancement of science and technology and the people's awareness of health, the incidence and mortality of gastric cancer have been controlled. However, this phenomenon is not optimistic.

Signal transducer and activator of transcription 3 (Stat 3 ) is a protein with dual functions of signal transduction and transcriptional regulation. Under normal circumstances, the body's Stat 3 exists in the cytoplasm in a non-activated state. When it is stimulated by the external environment, it can be activated into p-Stat3, and then enters the nucleus to bind to DNA, participate in the formation of tumor microenvironment by regulating downstream proinflammatory cytokines and factors that promote tumor growth and proliferation[3]. In recent years, many studies have shown that highly activated Stat 3 is found in various malignant tumor cells such as breast cancer, cervical cancer, prostate cancer, and oral squamous cell carcinoma. p-Stat3 can up-regulate the expression of downstream genes such as vascular endothelial growth factor (VEGF), cyclooxygenase 2 (COX-2) and metalloproteinases (MMPs), inhibit 
tumor cell apoptosis, promote tumor cell proliferation and neovascularization, as well as tumor cell infiltration and metastasis[4]

Among the cytokines induced by p-Stat3, Th17 cells and neutrophil secretion in the CD4+ T cell subset are the main sources of leukocyte 17 (Interleukin-17, IL-17). As a pro-inflammatory factor, IL-17 can not only promote tumor growth and metastasis, up-regulate the production of pro-angiogenic factors, but also play a clear role in tumors by enhancing the local T cell-associated immune response in tumors[5]. Some studies have shown that IL-17 expression enhanced in malignant tumors such as liver cancer, breast cancer, colorectal cancer, and lung cancer $[6,7,8]$. Some studies have shown that the expression levels of IL-17 in cancer tissues and serum of patients with gastric cancer are significantly higher than those in the normal groups, and IL-17A and IL-17F can be expressed in gastric cancer tissues and normal gastric mucosa tissues[9].

According to the Cell magazine, unless the microbe interacts with the immune system, the microbe itself cannot independently promote tumor growth. The bacterial metabolites can cause changes in the immune system, which in turn promotes the development and progression of tumors, and changes in the immune system can counteract microbes and thus affect tumors.Pathogen-Associated Molecular Patterns(PAMPs) can affect immune cells through Toll-like receptors and produce the inflammatory factor IL-17, which in turn activates the NF-KB/Stat3 signaling pathway and ultimately affects tumor progression[10]. Some studies showed that IL-17 affects the progression of chronic inflammations and tumors by promoting the release of various inflammatory factors, angiogenesis and cell proliferation[11].

The early symptoms of gastric cancer patients have no obvious specificity, which makes the diagnosis of early gastric cancer difficult, and it is easy to miss diagnosis and misdiagnosis. Therefore, how to improve the early diagnosis rate of gastric cancer, enhance the curative effect and prolong the survival time of patients is a difficult problem in clinical practice. By understanding the research status at home and abroad, we found that most scholar's research focuses on the changes of cytokines in different signaling pathways of different individual gastric cancers, and few people have studied the dynamic changes of gastric cancer progression in the same individual.

Therefore, our study collected and analyzed a large number of conventional gastroscope, then screened out a research population containing different stages of disease progression in the same individual and different stages of disease progression in different individuals. The immunohistochemical method was used to analyze the degree of activation of Stat3 and the expression of IL-17 in gastritis, GIN and gastric cancer.

\section{Patients And Methods}

\section{Patients}

We collected 70,137 cases of gastroscope data from November 2007 to December 2015 in the First Affiliated Hospital of Shihezi University School of Medicine. The pathological diagnosis was regarded as 
a standard, and the diagnosis sites and diagnosis results of all cases were all double-encoded. We excluded 5,790 patients with incomplete information or unclear diagnosis, and then compared the names, gender, ID number, telephone number, and home address of the remaining 64,347 patients.

Based on the number of patients' visits, the study population was divided into a single gastroscope group (54,846 cases) and gastroscope group (9,501 cases). We screened 36 patients with disease progression in multiple gastroscope populations, 21 of whom had intact paraffin tissue(Supplementary Table 1). We randomly selected gastritis (30 cases), intraepithelial neoplasia (30 cases) and gastric cancer (40 cases) from a single gastroscope group. A total of 100 cases were used as randomized control group, and 75 cases were reviewed as the final experimental sample (Supplementary Table 1).

All the cases were not treated with surgery, radiotherapy or chemotherapy. Each patient was diagnosed by at least two pathologists. The classification of gastritis level is based on the 2012 versionロConsensus opinion on chronic gastritis in Chinaland Gastric intraepithelial neoplasia and gastric cancer are diagnosed using the 2000 version of the WHO standard. Due to the small sample size, the specific subtypes of gastritis, GIN and GC are not further subdivided.

\section{Methods}

\section{Experimental methods}

All tissue specimens were fixed in $10 \%$ neutral formalin, embedded in paraffin, and sectioned thickness serially at 4um. Immunohistochemical staining was performed using the Envision two-step method. Specific antibodies: p-Stat 3 antibody (purchased from Abcam, ab76315, sourced from rabbit anti-human antibody, working concentration was 1:300, buffer was EDTA), IL-17 antibody (purchased from Abcam, ab79056, sourced from rabbit anti-human antibody, working concentration was 1:300, buffer was EDTA). The rest of the reagents were purchased from the Company of Beijing Zhongshan Jinqiao Biological. Breast cancer tissues stained positive with known p-Stat 3 and kidney cancer tissues positive for IL-17 staining were used as positive controls, respectively; PBS solution was used as a negative control.

\section{Results determination}

The results of the sectioning were judged by two pathologists using a double-blind method. The cytoplasm of IL-17 was positively expressed as light yellow to brown granules, and the nuclei of p-Stat3 was positively expressed as light yellow to brown granules. Ten different fields of view were randomly selected under a 400x optical microscope, and 100 cells of the same type were counted in each field of view. Immunohistochemistry results scoring criteria: A coloration intensity score: no coloration is 0 ; light yellow is 1 point; yellow is 2 points; brown yellow is 3 points; yellow is 4 points; $B$ the percentage of coloring cells number score: $0<5 \%$; 1 is $6 \%-25 \%$; 2 is $26 \%-50 \%$; 3 is $51 \%-75 \%$; 4 is $76 \%-100 \%$. The staining intensity score is multiplied by the percentage of stained cells number: $0-1$ is divided into $0 /(-), 2-4$ is divided into (1+), 5-8 is divided into (2+), 9-12 is divided ( $3+)$, and 13-16 is divided into (4+); negative expression is $0 /(-)$, weak positive expression is $(1+)$, and strong positive expression is $(2+-4+)$. 


\section{Statistical analyses}

Statistical analysis was performed using the SPSS statistical software package (version 17.0) $\llbracket$ KruskalWallis $\mathrm{H}$ test was adopted to compare the expression of IL-17 or p-Stat 3 in gastric cancer, intraepithelial neoplasia and gastritis; In random group and disease progression group, non-parametric test was used to compare the difference of IL-17 expression and p-Stat3 activation levels in different gastric diseases; Spearman rank correlation analysis was used to compare IL-17 expression and p-Stat3 activation level; Univariate logistic regression analysis of gender, age, IL -17 expression and p-Stat3 activation level were used to judge the hazards of gastric cancer; All $P$ values were bilateral, and difference with $P<0.05$ was considered statistically significant.

\section{Results}

Active p-Stat3 and IL-17 levels were tested using immunohistochemistry (IHC) assay in gastritis, GIN and GC

A positive stain for p-Stat3 was defined as light yellow or yellow-brown stain observed in the nucleus, IL17 with positive stain light yellow or yellow-brown is mainly localized in the cytoplasm. As shown in Figure 1, the coloration of degree and area were gradually increased in gastritis, GIN and GCdegree of pStat3 activation and IL-17 levels.

\section{The degree of p-Stat3 activation and the expression of IL-17 in gastritis, GIN and GC}

In the random group and the progression group of Table 1, the degree of p-Stat3 activation showed that the strong positive expression rate in the three groups between gastritis, GIN and GC gradually increased with the severity of the disease, and the difference was statistically significant (random group $\chi^{2}=52.99$, $P<0.001$; progression group $\left.\chi^{2}=28.57, P<0.001\right)$, and the degree of $p-S t a t 3$ activation was the highest in gastric cancer tissues. There were differences statistically significant of the expression of IL-17 levels in both group, and the expression rate was also increased with disease severity among the gastritis, GIN and GC. $\otimes$ the random group $\chi^{2}=45.29 \varangle P<0.001 \rrbracket$ the progress group $\chi^{2}=24.02 \varangle P<0.001 \rrbracket$, the higher expression rate of IL-17 appeared on the gastric cancer between the both groups (Table 2) .

\section{The differences between p-Stat3 activation and IL-17 expression in random and progression groups in different diseases}

In Table 3, the degree of p-Stat3 activation and IL-17 expression in the randomized and advanced groups were not different in gastritis, gastric epithelial neoplasia, and gastric cancer tissues, $p>0.05$. This suggests that p-Stat3 and IL-17 are persistent in different stages of gastritis-gastric intraepithelial neoplasia-gastric cancer progression, a universal phenomenon but not specific.

\section{The degree of p-Stat3 activation and the expression of IL-17 in gastritis, GIN and GC}


AS there was no significant difference in the degree of p-Stat3 activation and IL-17 expression between gastritis, GIN and GC in the randomized group and the progressive group, sothedata oftwo groups were combined. In Figure 2, the mean \pm standard deviation $(M \pm S D)$ of $p$-Stat3 activation in gastritis was $0.53 \pm 1.11,4.21 \pm 2.59$ in GIN, and $8.62 \pm 2.83$ in GC. The mean \pm standard deviation (M $\pm S D)$ of IL-17 in gastritis was $2.97 \pm 2.02,8.07 \pm 3.04$ in $\mathrm{GIN}$, and $11.51 \pm 2.94$ in GC. The degree of p-Stat3 activation and IL17 expression were different in gastritis and GIN, GIN and GC, gastritis and GC. It wasverified that the degree of $\mathrm{p}$-Stat3 activation and IL-17 expression differed at various stages of the disease again .

\section{Using ROC curve to analyze the ability of each index to predict gastric cancer}

The area under the showss the ability of each indicator to predict gastric cancer. The larger the area under the curve is, the stronger the predictive abilityis. The Yoden index is a comprehensive index for evaluating sensitivity andspecificity. Correspondingly, the larger the area under the curveis, the higher the Yoden indexis. In Fig. 3, the area under thecurveof p-Stat3 is the largest $(0.923, \mathrm{P}<0.001)$, the maximum value of the Yoden index is 0.707 , and the cut-off value is 5 . The area under thecurveofIL-17 line is $0.844(P<$ 0.001 ), the maximum value of the Yoden index is 0.615 , and the cut-off value is 7 . Age and gender did not show significant predictive power in this study.

\section{Logistic analysis of risk factors for gastric cancer}

According to the cut-off value calculated by ROC as the critical value, the value of the cut-off value is greater than the critical value of the risk of gastric canceror equal, and is less than the critical valueregardedas the low risk group of gastric cancer. According to the results in Table 4, high activation levels of $\mathrm{p}$-Stat 3 and high expression of IL-17 bothare risk factors for gastric cancer.

\section{Discussion}

The signaling and transcriptional activator Stat3 is an important member of the Stat protein family and is a molecular protein with cell signaling and activation of gene transcriptions. Under normal circumstances, a small amount of Stat 3 molecules exist in the body and exist in the non-activated state outside the cells. When stimulated by the outside world, it can bind to the corresponding receptor on the cell membrane through cytokines such as extracellular signal proteins TGF-, IL-23, IL-21, and IL-6. In the cytoplasm, Jak tyrosine kinase mediated Stat3 phosphorylation, phosphorylated Stat3 enters the activated state, $p$-Stat3 enters the nucleus as a homodimer, regulateing downstream specific target genes and affects body.

The results of immunohistochemistry in this study showed that p-Stat 3 was activated in gastritis tissue with little or no obvious expression; p-Stat3 was expressed in the nucleus of gastric glandular cells in intraepithelial neoplasia; In gastric cancer tissues, the degree of activation inp-Stat3 is stronger than that of gastric epithelial neoplastic tissue, and the coloration area is larger. This phenomenon exists in both randomized and disease-promoting populations. In the randomized group, the positive expression rates of activated p-stat3 in gastritis, gastric intraepithelial neoplasia and gastric cancer tissues were $23.8 \%$, 
$85.7 \%$ and $92.3 \%$, among which the strong positive expression rates were $4.8 \%, 42.9 \%$ and $69.2 \%$.In the disease progression group, the positive rate of p-Stat3 was $17.6 \%$ in gastritis, $93.3 \%$ in gastric epithelial tumors, and $100 \%$ in gastric cancer tissues. The strong positive expression rates were $5.9 \%, 13.3 \%$, and $81.8 \%$. The positive expression rate of $\mathrm{p}$-Stat 3 in the randomized group and the disease progression group gradually increased with the progression of the disease, and the difference was significant $(P<0.001)$, suggesting that the abnormal activation of $p$-Stat 3 may be closely related to the occurrence of gastric cancer.

In human gastric cancer and colorectal cancer studies, high-density Th17 cells are associated with increased angiogenesis in invasive tumors[12,13]. Tumors with high levels of IL-17mRNA, the number of vascular endothelial cells and infiltrating neutrophils was significantly higher than the low one[12]. All above suggest that IL-17 high expression is involved in tumor angiogenesis and promotes tumor growth. There are many ways of tumor metastasis. The common ones are blood and lymphatic metastasis. IL-17 in non-small cell lung cancer tumors can induce the production of VEGF-C and VEGF-D, which is associated with increased lymphangiogenesis and poor prognosis[14]. The expression levels of IL-17 in serum and cancer tissues of patients with gastric cancer were significantly higher than those in the normal group. What's more, IL-17A and IL-17F could also promote the proliferation of gastric cancer cell lines[15,16]. With the progression of the lesion, the positive expression rate of IL-17 is gradually increased in cervical control tissues, CIN1, CIN2, CIN3 and cervical squamous cell carcinoma tissues[17], which can be inferred that IL-17 is in tumor growth, metastasis and angiogenesis play a role.

The results of immunohistochemistry in randomized and advanced populations of this study confirmed that IL-17 expression was low in gastritis tissues; IL-17 is only expressed in the cytoplasm of gastric glandular cells of intraepithelial neoplasia, and its staining intensity is significantly deeper than that of gastritis; In gastric cancer tissues, IL-17 expression was observed in the cytoplasm of gastric cancer cells, monocytes and vascular epithelial cells. Compared with the intraepithelial neoplasia tissue, the expression level of IL-17 was significantly increased in gastric cancer tissues, and the coloration was deeper and the area was larger, which indicated that both the random group and the disease progression population existed. In the randomized group, the positive expression rates of IL-17 in gastritis, gastric intraepithelial neoplasia and gastric cancer were respectively $76.2 \%, 100 \%$, and $100 \%$, respectively, and the strong positive expression rates were respectively $19.0 \%, 89.2 \%$, and $84.6 \%$. In the disease progression group, the positive expression rates of IL-17 in gastritis, gastric intraepithelial neoplasia and gastric cancer were respectively $88.2 \%, 100 \%$, and $100 \%$, and the strong positive expression rates were respectively $17.6 \%, 80.0 \%$, and $100 \%$. The positive expression rate of IL-17 in the randomized group and the disease progression group gradually increased with the progression of the lesion, and the difference was significant at different stages $(P<0.001)$. By further comparing the expression of IL-17 in gastritis, gastric intraepithelial neoplasia and gastric cancer tissues in the randomized and advanced groups, the results showed no significant difference between the two groups $(P>0.05)$. It is suggests that the abnormal expression of IL-17 may be an early event of gastric cancer, which plays a role in promoting the progression from gastritis to gastric intraepithelial neoplasia to the development of gastric cancer. In the early stage of gastritis, the immune system secretes a small amount of IL-17. It synergizes with various 
cytokines to amplify the inflammatory response, and damages the gastric mucosa leading to disease progression. With the progression of the disease, the injured gastric tissue further recruits the autoimmune system and the adaptive immune system secretes more IL-17, which induces the mutation of cell damage gene and leads to the occurrence of tumor. This is a common phenomenon and does not produce different results due to individual differences.

Th17 differentiation is impaired in CD4+ T cells of Stat3-deficient mice, whereas overexpression of $p$ Stat3 increases IL-17 production[18,19]. Stat3 is highly activated and IL-17 is highly expressed in colon and breast cancer[20,21]. In this study, IL-17 expression, Stat3 activation was highly positively correlated with disease severity. As the disease progresses, the degree of Stat 3 activation is low when IL-17 is low, and the level of Stat 3 is also increased when IL-17 is high. Both are consistent in gastric tissue and are expressed in gastric cancer. The highest, followed by intraepithelial neoplasia, the lowest gastritis.

In summary, Stat3 activation level and IL-17 expression may be involved in the development and metastasis of gastric cancer, suggesting that p-Stat3 and IL-17 play important roles in carcinogenesis and cancer progression, providing new ideas for targeted treatment of gastric cancer. At the same time, it's impact on the survival of gastric cancer patients suggests that p-Stat3 and IL-17 may also be used as reference indicators for the prognosis of gastric cancer patients.

\section{Conclusion}

With the disease increasingly serious, the Stat3 activation level and IL-17 expression are higher. There is a high correlation between activated Stat3 and IL-17 expression, both of which are risk factors for gastric cancer. This study provided a theoretical basis for further clarifying the influence of p-stat 3 and il-17 pathways on the occurrence, development and prognosis of gastric cancer, so as to provide a more reliable theoretical basis for the prevention, diagnosis and treatment of gastric cancer.

\section{Abbreviations}

GIN: gastric intraepithelial neoplasia; GC: gastric cancer.

\section{Declarations}

\section{Acknowledgements}

None.

Author's contributions: Planning and execution of the study: LJW, CC, XJW, LT, FL and WJZ. Patient recruitment/data collection: LJW, CC, XJW and LT. Manuscript drafting: LJW and WJZ. Help to analyze the data: LJW Critical editing and final approval of the manuscript: WJZ and LJW. All authors have approved the final draft. 


\section{Funding}

This work was supported by grants from the Ministry of Science and Technology of China (nos. 2009BAI82B02 and 2010DFB34100) and the Oasis Scholar Fund of Shihezi University (no. LZXZ201023). The funders had no role in study design, data collection and analysis, decision to publish or preparation of the manuscript.

\section{Availability of data and materials}

The datasets during and/or analyzed during the current study available from the corresponding author on reasonable request.

\section{Ethics approval and consent to participate}

Ethical approval was obtained from the Institutional Ethics Review Board (IERB) of the First Affiliated Hospital of School of Medicine, Shihezi University (No. 2018-067-01). The IERB waived the need for patient consents due to anonymous analyses of the data and confidentiality and anonymity in the handling and publication of patients' tissues. Standard University Hospital Guidelines in accordance with the Declaration of Helsinki were followed in this study.

\section{Consent for publication}

Not applicable.

\section{Competing interests}

The authors declare that they have no competing interests.

\section{References}

[1] F. Bray, J. Ferlay, I. Soerjomataram, R.L. Siegel, L.A. Torre, A. Jemal, Global cancer statistics 2018: GLOBOCAN estimates of incidence and mortality worldwide for 36 cancers in 185 countries, CA Cancer J Clin 68 (2018) 394-424.

[2] W. Chen, R. Zheng, P.D. Baade, S. Zhang, H. Zeng, F. Bray, A. Jemal, X.Q. Yu, J. He, Cancer statistics in China, 2015, CA Cancer J Clin 66 (2016) 115-132.

[3] S.I. Grivennikov, F.R. Greten, M. Karin, Immunity, inflammation, and cancer, Cell 140 (2010) 883-899.

[4] J.Y. Yoo, W.S. Yang, J.H. Lee, B.G. Kim, R.R. Broaddus, J.M. Lim, T.H. Kim, J.W. Jeong, MIG-6 negatively regulates STAT3 phosphorylation in uterine epithelial cells, Oncogene 37 (2018) 255-262.

[5] M. Numasaki, J. Fukushi, M. Ono, S.K. Narula, P.J. Zavodny, T. Kudo, P.D. Robbins, H. Tahara, M.T. Lotze, Interleukin-17 promotes angiogenesis and tumor growth, Blood 101 (2003) 2620-2627. 
[6] S.I. Grivennikov, K. Wang, D. Mucida, C.A. Stewart, B. Schnabl, D. Jauch, K. Taniguchi, G.Y. Yu, C.H. Osterreicher, K.E. Hung, C. Datz, Y. Feng, E.R. Fearon, M. Oukka, L. Tessarollo, V. Coppola, F. Yarovinsky, H. Cheroutre, L. Eckmann, G. Trinchieri, M. Karin, Adenoma-linked barrier defects and microbial products drive IL-23/IL-17-mediated tumour growth, Nature 491 (2012) 254-258.

[7] J.W. Du, K.Y. Xu, L.Y. Fang, X.L. Qi, Interleukin-17, produced by lymphocytes, promotes tumor growth and angiogenesis in a mouse model of breast cancer, Mol Med Rep 6 (2012) 1099-1102.

[8] G.Q. Zhang, F. Han, X.Z. Fang, X.M. Ma, CD4+, IL17 and Foxp3 expression in different pTNM stages of operable non-small cell lung cancer and effects on disease prognosis, Asian Pac J Cancer Prev 13 (2012) 3955-3960.

[9] J. Li, G.K. Lau, L. Chen, S.S. Dong, H.Y. Lan, X.R. Huang, Y. Li, J.M. Luk, Y.F. Yuan, X.Y. Guan, Interleukin 17A promotes hepatocellular carcinoma metastasis via NF-kB induced matrix metalloproteinases 2 and 9 expression, PLoS One 6 (2011) e21816.

[10] N. Gagliani, B. Hu, S. Huber, E. Elinav, R.A. Flavell, The fire within: microbes inflame tumors, Cell 157 (2014) 776-783.

[11] J. Mudter, M.F. Neurath, II-6 signaling in inflammatory bowel disease: pathophysiological role and clinical relevance, Inflamm Bowel Dis 13 (2007) 1016-1023.

[12] T. lida, M. Iwahashi, M. Katsuda, K. Ishida, M. Nakamori, M. Nakamura, T. Naka, T. Ojima, K. Ueda, K. Hayata, Y. Nakamura, H. Yamaue, Tumor-infiltrating CD4+ Th17 cells produce IL-17 in tumor microenvironment and promote tumor progression in human gastric cancer, Oncol Rep 25 (2011) 12711277.

[13] J. Liu, Y. Duan, X. Cheng, X. Chen, W. Xie, H. Long, Z. Lin, B. Zhu, IL-17 is associated with poor prognosis and promotes angiogenesis via stimulating VEGF production of cancer cells in colorectal carcinoma, Biochem Biophys Res Commun 407 (2011) 348-354.

[14] S.K. Chauhan, Y. Jin, S. Goyal, H.S. Lee, T.A. Fuchsluger, H.K. Lee, R. Dana, A novel prolymphangiogenic function for Th17/IL-17, Blood 118 (2011) 4630-4634.

[15] Y. Yamada, H. Saito, M. Ikeguchi, Prevalence and clinical relevance of Th17 cells in patients with gastric cancer, J Surg Res 178 (2012) 685-691.

[16] X. Wu, Z. Zeng, B. Chen, J. Yu, L. Xue, Y. Hao, M. Chen, J.J. Sung, P. Hu, Association between polymorphisms in interleukin-17A and interleukin-17F genes and risks of gastric cancer, Int J Cancer 127 (2010) 86-92.

[17] S.S. Devesa, E.L. Diamond, Association of breast cancer and cervical cancer incidence with income and education among whites and blacks, J Natl Cancer Inst 65 (1980) 515-528. 
[18] X.O. Yang, A.D. Panopoulos, R. Nurieva, S.H. Chang, D. Wang, S.S. Watowich, C. Dong, STAT3 regulates cytokine-mediated generation of inflammatory helper T cells, J Biol Chem 282 (2007) 93589363.

[19] T.J. Harris, J.F. Grosso, H.R. Yen, H. Xin, M. Kortylewski, E. Albesiano, E.L. Hipkiss, D. Getnet, M.V. Goldberg, C.H. Maris, F. Housseau, H. Yu, D.M. Pardoll, C.G. Drake, Cutting edge: An in vivo requirement for STAT3 signaling in TH17 development and TH17-dependent autoimmunity, J Immunol 179 (2007) 43134317.

[20] Y.X. Li, L. Zhang, D. Simayi, N. Zhang, L. Tao, L. Yang, J. Zhao, Y.Z. Chen, F. Li, W.J. Zhang, Human papillomavirus infection correlates with inflammatory Stat3 signaling activity and IL-17 level in patients with colorectal cancer, PLoS One 10 (2015) e0118391.

[21] N. Zhang, Z.P. Ma, J. Wang, H.L. Bai, Y.X. Li, Q. Sun, L. Yang, L. Tao, J. Zhao, Y.W. Cao, F. Li, W.J. Zhang, Human papillomavirus infection correlates with inflammatory Stat3 signaling activity and IL-17 expression in patients with breast cancer, Am J Transl Res 8 (2016) 3214-3226.

\section{Tables}

Table 1 Different levels of p-Stat3 activation in gastritis, GIN and GC

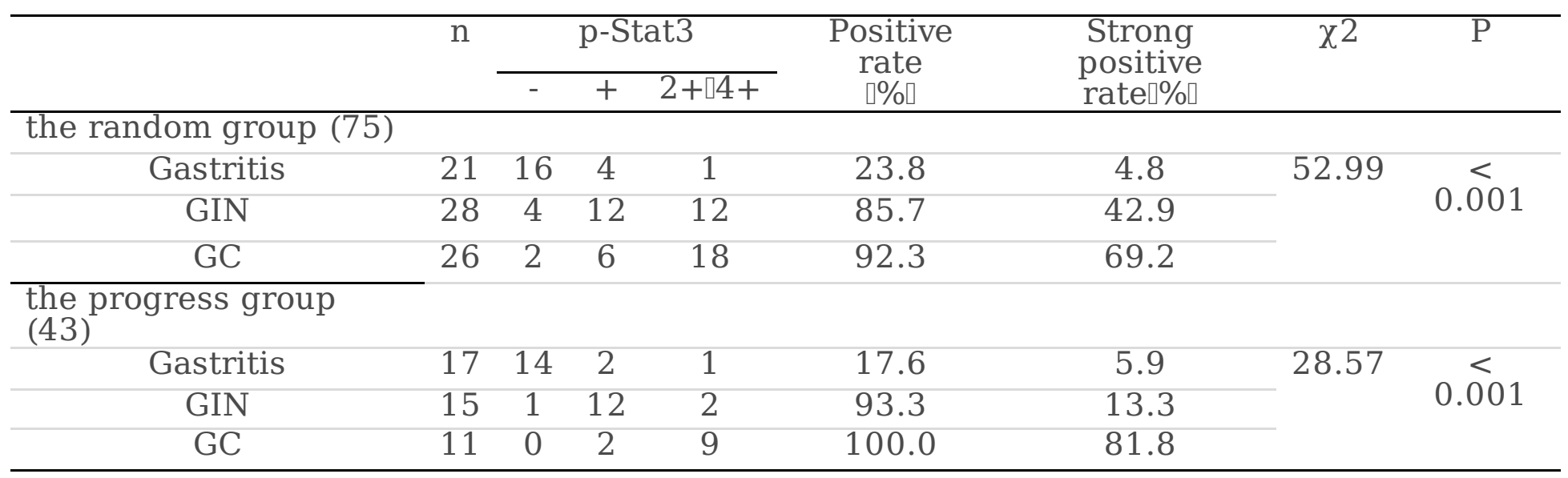

Note: GIN = Gastric Intraepithelial Neoplasia. GC $=$ Gastric Cancer

Table 2 Different levels of IL-17 expression in gastritis, GIN and GC 

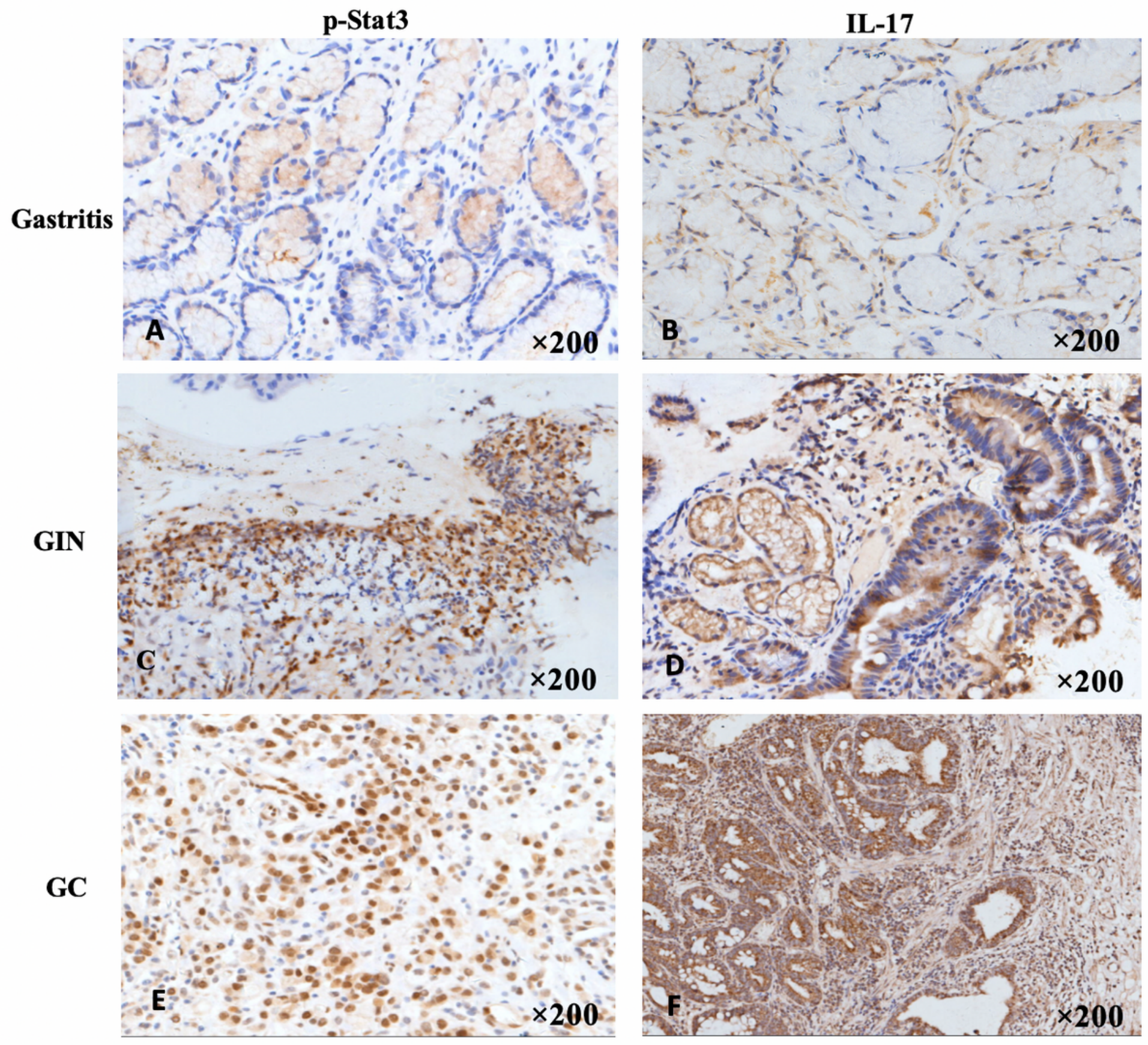

\section{Figure 1}

Activation of p-Stat3 and expression of IL-17 in gastritis, GIN and GC Figure Legends: The negative staining of $p$-Stat3 in gastritis tissues $(\times 200)$; B the positive staining of $p$-Stat3 in GIN tissues $(\times 200)$; C the positive staining of p-Stat3 in GC tissue $\nabla \times 200$ ); D the negative staining of IL-17 in gastritis tissues ( $\times 200)$; $E$ the positive staining of IL-17 in GIN tissues (×200); $F$ the positive staining of IL -17 in GC tissue $\nabla \times 200)$. 


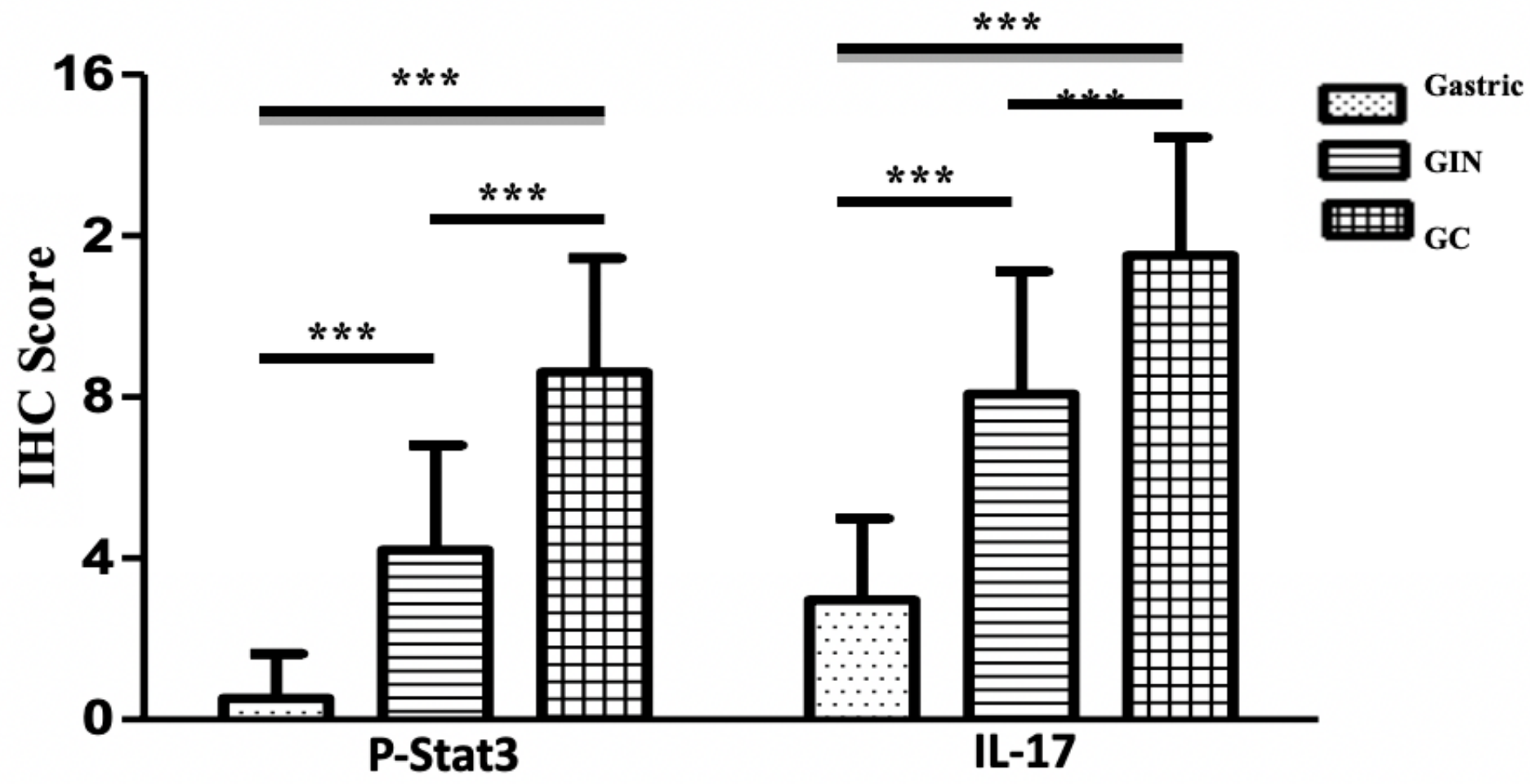

Note: ${ }^{* * *}$ represent $\mathrm{P}<0.001$

Figure 2

Differences in p-Stat3 activation and IL-17 expression levels in gastritis, GIN and GC 


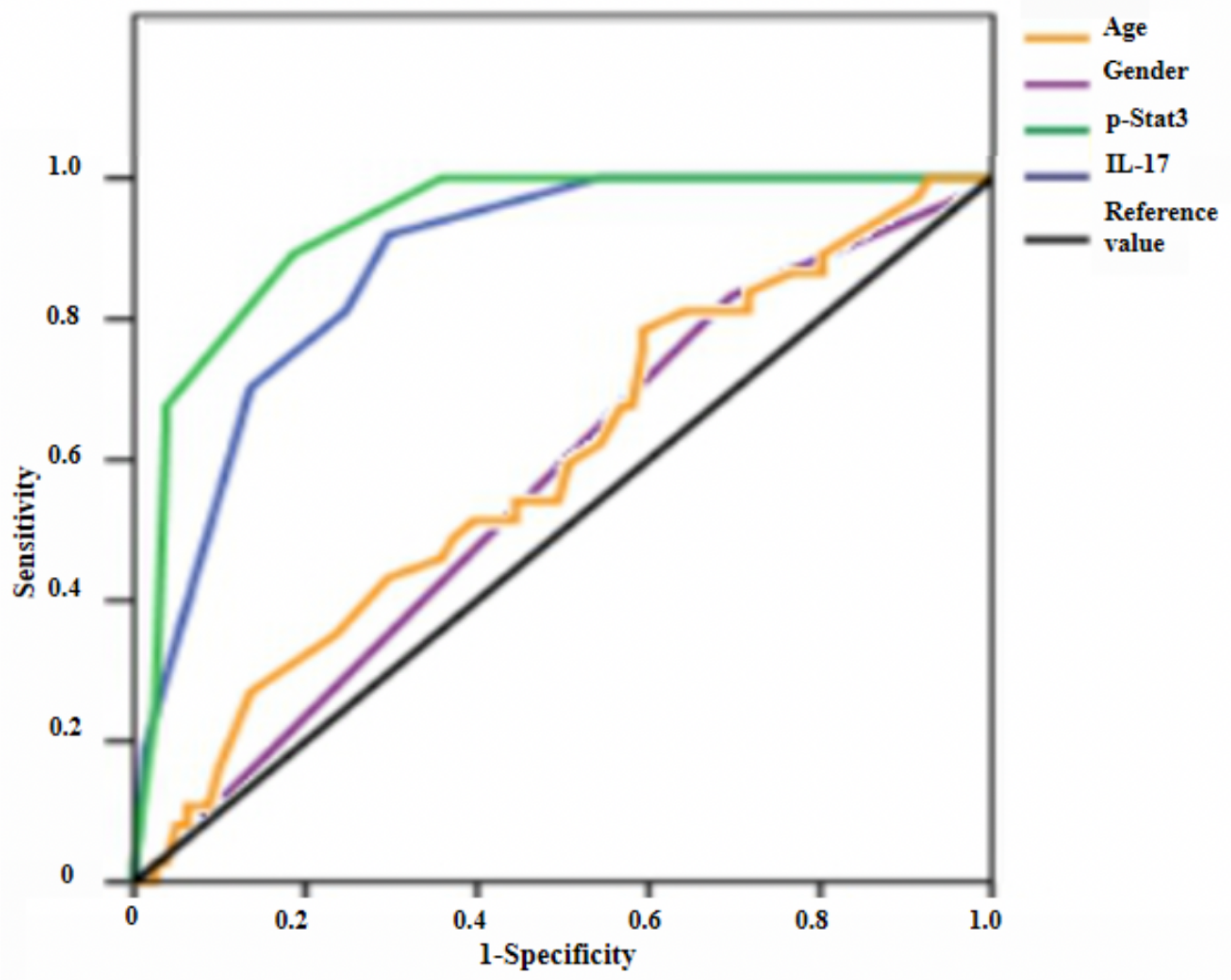

Figure 3

ROC curve shows the ability of each indicator to predict GC

\section{Supplementary Files}

This is a list of supplementary files associated with this preprint. Click to download.

- 4.SupplementaryMaterial.doc 